\title{
DC electrical conduction in heat-treated poly nickel phthalocyanine
}

\author{
B SANJAI, ANASUYA RAGHUNATHAN, T S NATARAJAN*, \\ G RANGARAJAN and S VENKATACHALAM \\ Department of Physics, Indian Institute of Technology, Madras 600036, India \\ 'Polymers and Special Chemicals Division, VSSC, Trivandrum 695022, India \\ MS received 16 July 1996; revised 21 November 1996
}

\begin{abstract}
Heat treatment of poly nickel phthalocyanine leads to a charge transfer and hence there is an improvement in conductivity by 5 orders of magnitude. DC electrical conductivity measurements have been carried out in the heat-treated samples in the temperature range $270 \mathrm{~K}$ $10 \mathrm{~K}$. Mott's variable range hopping conduction in one dimension is observed in the temperature range $270 \mathrm{~K}-77 \mathrm{~K}$ and the conductivity remains constant in the temperature range $77 \mathrm{~K}-10 \mathrm{~K}$.
\end{abstract}

Keywords. Conducting polymers; phthalocyanine; conductivity; variable range hopping.

\section{Introduction}

The mechanism of electrical conduction in conducting polymers has been of interest since the report of the successful $p$ and $n$ doping of polyacetylene to the metallic state by Shirakawa et al (1977). Tsukomoto et al (1990) found it possible to achieve metal-like conductivity by the addition of electron donors or electron acceptors to polyacetylene. Recently Reghu et al (1992) protonated polyaniline with camphor sulphonic acid and found that this showed a metal-insulator transition. Most of the conducting polymers are unstable under ambient conditions and there is a degradation of electrical, thermal, mechanical and chemical properties upon doping. Poly metallo phthalocyanines have gained considerable interest because of their stability against heat, light, moisture and air and they show an increase in conductivity by 5 orders of magnitude upon heat treatment. Phthalocyanines have a sheet-like structure and have been studied extensively by Schramm et al (1980). They are useful for applications in molecular electronic devices.

Heat-treated copper phthalocyanine shows a three dimensional variable range hopping conduction in the temperature range $270 \mathrm{~K}-35 \mathrm{~K}$ and later Raghunathan et al (1991) reported fluctuation induced tunneling conduction when the temperature range was extended to $4.2 \mathrm{~K}$. For the case of heat treated cobalt phthalocyanines, a three dimensional variable range hopping conduction was observed by Sanjai et al (1995). A two-dimensional variable range hopping conduction was observed for heat-treated nickel phthalocyanines in the temperature range $270 \mathrm{~K}-200 \mathrm{~K}$ by Anasuya et al (1991). In the present work, temperature-dependent DC electrical conductivity has been studied on heat-treated nickel phthalocyanines in the temperature range $300 \mathrm{~K}-15 \mathrm{~K}$ to understand the exact details of the conduction mechanism.

\section{Synthesis and experimental}

I[NiPc $]_{n}$ was prepared by the method of Achar et al (1982). Analysis for anhydrideterminated polymer $\left(\mathrm{C}_{120} \mathrm{H}_{24} \mathrm{~N}_{32} \mathrm{O}_{24} \mathrm{Ni}_{4}\right)$ : Calculated C, 56.9; $\mathrm{H}, 0.95 ; \mathrm{N}, 17 \cdot 7 ; \mathrm{Ni}, 9 \cdot 22$.

*Author for correspondence 
Found $\mathrm{C}, 56 \cdot 1 ; \mathrm{H}, 1 \cdot 12 ; \mathrm{N}, 17 \cdot 9 ; \mathrm{Ni}, 9 \cdot 3$. The prepared tetramer was purified, characterized and converted to the polymer II $[\mathrm{NiPc}]_{n}$ by the heat treatment procedure described below as reported earlier by Venkatachalam et al (1988).

Polymer I was pressed into pellets of about $13 \mathrm{~mm}$ diameter and $1 \mathrm{~mm}$ thickness under 8-10 tons of load in Carver press. The pellet was heated in helium (flow rate $50 \mathrm{ml} / \mathrm{min}$ ) at a rate of $20^{\circ} \mathrm{C} / \mathrm{min}$ from $298 \mathrm{~K}$ to $493 \mathrm{~K}$. After holding the pellet at $473 \mathrm{~K}$ for $15 \mathrm{~min}$, the heating was continued at the same rate till the temperature attained $753 \mathrm{~K}$. The pellet was kept at this temperature for $45 \mathrm{~min}$ and then cooled to room temperature in a helium atmosphere. The product obtained is referred to as polymer II. The pellet was weighed before and after the heat treatment. The weight loss observed was $40 \%$. Analysis for polymer II $\left(\mathrm{C}_{104} \mathrm{H}_{24} \mathrm{~N}_{32} \mathrm{Ni}_{4}\right)$ was: Calculated (taking into account $9 \%$ adsorbed oxygen) C, 58.09; H, 1.12; N, 20.84; Ni, 10.92 and found C, 57.4; H, 1.1; N, 20.1; Ni, $10 \cdot 6$.

Samples, as above-formed pellets, were used for DC electrical conductivity measurements. A two-probe method was used for the conductivity measurements. A constant current was applied from a Keithley 225 current source. The voltage was measured using a Keithley 617 electrometer. Gold was thermally evaporated on both sides of the pellets for making contacts. A dip-stick arrangement was used in a helium bath type cryostat for these measurements. The temperature was measured using a calibrated $\mathrm{Au}(0.07 \%)-\mathrm{Fe}$-chromel thermocouple.

\section{Results}

The DC electrical conductivity of I $[\mathrm{NiPc}]_{n}$ at $270 \mathrm{~K}$ was $5 \times 10^{-8} \mathrm{~S} / \mathrm{cm}$. Upon heat treatment the value of conductivity of II $[\mathrm{NiPc}]_{n}$ increased to $5 \times 10^{-3} \mathrm{~S} / \mathrm{cm}$. The

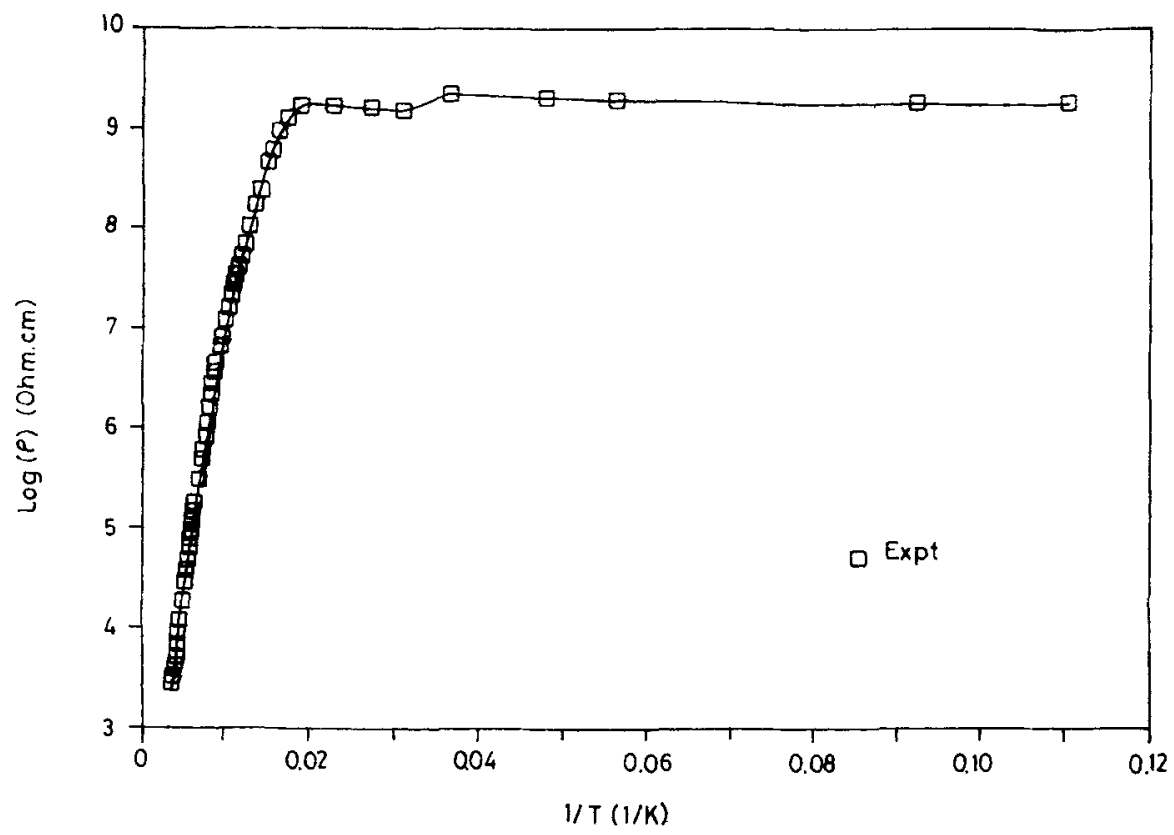

Figure 1. A plot of $\log (\rho)$ vs $1 / T$ in the temperature range $270 \mathrm{~K}$ to $10 \mathrm{~K}$. The resistivity increases rapidly up to $77 \mathrm{~K}$ and remains constant below $77 \mathrm{~K}$ down to $10 \mathrm{~K}$. The squares show the experimental data points and the solid line is simply the line joining points. 


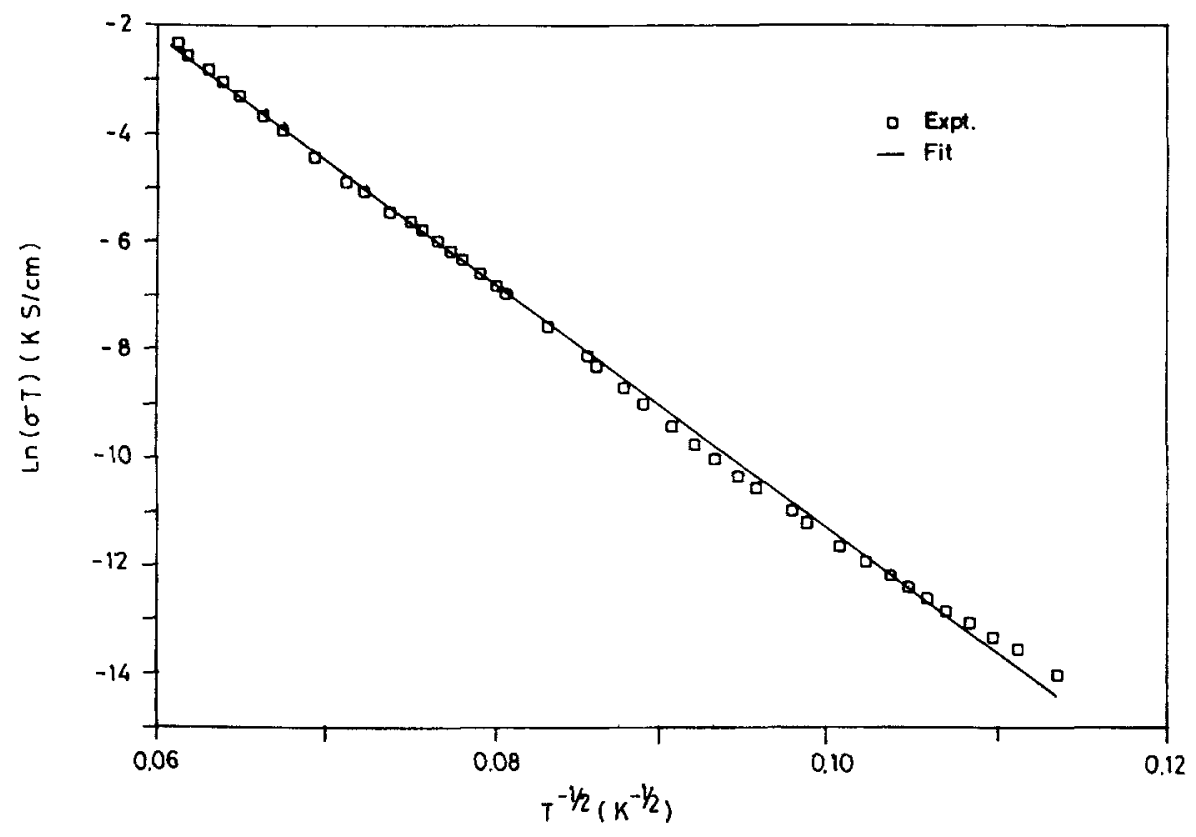

Figure 2. A plot of $\operatorname{Ln}(\sigma \cdot T)$ vs $T^{-1 / 2}$ in the temperature range $270 \mathrm{~K}$ to $77 \mathrm{~K}$. The squares are the experimental data points and the solid line shows a fit corresponding to one dimensional variable range hopping.

corresponding value for II [CuPc $]_{n}$ reported earlier was more than this value by 2 orders of magnitude. In the temperature range $270 \mathrm{~K}-77 \mathrm{~K}$ the best fit for the conductivity could be obtained (as shown in figure 2) to an expression of the form

$$
\sigma(T)=\left(\sigma_{0} / T\right) \exp \left[-\left(T_{0} / T\right)^{1 / 2}\right]
$$

where $\sigma$ is the conductivity at a temperature $T$ and $T_{0}$ the Mott's characteristic temperature. In the above equation $\sigma \rightarrow \sigma_{0}$ when $T \rightarrow T_{0}$. The above expression is characteristic of Mott's variable range hopping in one dimension (1D-VRH) (Mott and Davis 1979). The value of $T_{0}$ for II $[\mathrm{NiPc}]_{n}$ is $52,267 \mathrm{~K}$. The sample showed an ohmic behaviour up to a field of $1000 \mathrm{~V} / \mathrm{cm}$. The value of conductivity remained a constant between $77 \mathrm{~K}$ and $10 \mathrm{~K}$ as shown in the plot between $\log (\rho)$ and $1 / T$ in figure 1 .

\section{Discussion}

In II [CuPc $]_{n}$, it has been reported by Raghunathan et al (1991) that conduction occurred by electron transfer across insulating gaps in conducting pathways by fluctuation-induced tunneling model, proposed by Sheng (1980). The metallic regions corresponded to the crystalline or more ordered regions of the polymer and the amorphous or less ordered regions to the insulating regions. Such a model is valid only when the size of conducting regions are large as compared to the insulating barrier width. In the case of $\mathrm{II}[\mathrm{NiPc}]_{n}$, a 1D-VRH conduction was observed. This may be because, the crystalline or more ordered regions could have been small in size separated by large or less-ordered regions where hopping conduction dominates. X-ray diffraction of II $[\mathrm{NiPc}]_{n}$ by Raghunathan (1994) 
showed the presence of some peaks corresponding to an interplanar spacing of $3.06 \AA$. This model is corroborated by Wang et al (1992) who states that conduction is due to hopping at the localized states in the amorphous regions between the small metallic regions. The more dominant contribution is reflected in the temperature-dependent DC conductivity.

This mechanism of conduction is also supported by the temperature-dependent DC magnetic susceptibility $(\kappa)$ and thermopower measurements of Anasuya et al (1991). According to them the value of $\kappa$ shows a transition from diamagnetic to paramagnetic upon heat treatment and the sign of thermopower shows the presence of $p$ type charge carriers. Hence heat treatment leads to a charge transfer, resulting in an incompletely filled band. The value of Pauli contribution to paramagnetism at room temperature has been reported to be $1.2 \times 10^{-5} \mathrm{emu} / \mathrm{g}$. A linear temperature dependence of thermopower also indicates the presence of metallic regions in the material.

\section{Conclusions}

Heat treatment of I[NiPc $]_{n}$ leads to an enhancement in the conductivity by 5 orders of magnitude. Conduction in II $[\mathrm{NiPc}]_{n}$ takes place by hopping between the localized states in the amorphous regions between the conducting small metallic regions. A fit to Mott's 1D-VRH model could be obtained for the variation of conductivity in the temperature range $270 \mathrm{~K}$ to $77 \mathrm{~K}$. From $77 \mathrm{~K}$ to $10 \mathrm{~K}$ the value of conductivity remains a constant.

\section{References}

Achar B N, Fohlen B M and Parker B A 1982 J. Polym. Sci. Polym. Chem. 201785

Anasuya M V, Natarajan T S, Rangarajan G, Venkatachalam S and Manoharan P T 1991 Solid State Commun. 77661

Mott N F and Davis E A 1979 Electronic processes in non-crystalline materials (London: Oxford University Press) Raghunathan Anasuya 1994 Charge transport, magnetic and optical properties of some conducting polymers, Ph. D. thesis, IIT, Madras

Raghunathan Anasuya, Natarajan T S and Rangarajan G 1991 Phys. Lett. A159 89

Reghu M, Cao Y, Moses D and Heeger A J 1992 Phys. Rev. B47 1758

Sanjai B, Anasuya Raghunathan, Natarajan T S and Rangarajan G 1995 Mater. Sci. \& Eng. C3 227

Schramm Charles J, Scaringe Raymond P, Stojkovic Djordje R, Hoffmann Brian M, Ibers James A and Marks Tobin J 1980 J. Am. Chem. Soc. 1026703

Sheng Ping 1980 Phys. Rev. B21 2180

Shirakawa H, Louis E J, MacDiarmid A G, Chiang C K and Heeger A J 1977 J. Chem. Soc. Chem. Commun. 57 578

Tsukomoto Jun, Takahashi Akio and Kawasaki Kikuko 1990 Jap. J. Appl. Phys. 29125

Venkatachalam S, Rao K V C and Manoharan P T 1988 Synth. Met. 26237

Wang Z H, Scherr E M, MacDiarmid A G and Epstein A J 1992 Phys. Rev. B45 4190 